\title{
Comorbidades e riscos à mortalidade dos idosos pós-cirurgia de fratura de quadril: uma revisão integrativa da literatura
}

\author{
Comorbidities and risks to mortality of elderly after hip fracture surgery: an integrative \\ literature review
}
Comorbilidades y riesgos para la mortalidad de personas mayores después de la cirugía de fractura de cadera: una revisión integrativa de la literatura

Allex Alves Sobral de Sousa ${ }^{1 *}$, Lucineide Coqueiro Gurgel ${ }^{1}$, Carmelita Maria Silva Sousa ${ }^{1}$, Eulina Alves Sousa Brito ${ }^{1}$, Iasmin Belém Silva Queiroz ${ }^{1}$, Maria Aparecida Feitosa Cândido Herculano ${ }^{1}$, Lina Maria Vidal Romão ${ }^{1}$, Cícera Alves de Luna ${ }^{1}$, Dayse Christina Rodrigues Pereira Luz², Willma José de Santana².

\section{RESUMO}

Objetivo: Realizar um levantamento na literatura para determina os efeitos das comorbidades na recuperação dos idosos pós-cirurgia de fratura de quadril e os riscos à mortalidade deste paciente. Métodos: Trata-se de uma revisão integrativa de abordagem qualitativa. Realizada nas seguintes bases de dados LILACS, MEDLINE, SCIELO e PUBMED, utilizando-se dos seguintes Descritores em Ciências da Saúde (decs): Quedas; Idosos; Fraturas do quadril; Comorbidade e Mortalidade. Resultados: $\mathrm{Na}$ análise dos estudos verificou-se que a maior frequência de quedas e fraturas de quadril está entre as mulheres idosas, sendo que o período pós-cirúrgico está relacionado ao surgimento de comorbidades referentes com o déficit nas funções física, dor corporal e medo de novas quedas. Pacientes que permanecem por mais tempo internados após a cirurgia possuem maior chance ao aparecimento de comorbidades, comprometendo sua recuperação, e assim, proporcionando maiores riscos a mortalidade prematura do idoso. Considerações Finais: Os pacientes com fraturas do quadril e que apresentam comorbidades no pós-cirúrgico, estão predispostos a intercorrências clínicas e têm uma taxa de mortalidade elevada. Neste sentido, existe a necessidade de se elaborar ações que garanta a proteção e a recuperação deste idoso, de forma a diminuir os riscos a mortalidade após fratura de quadril.

Palavras-chave: Quedas, Idosos, Fraturas do quadril, Comorbidade, Mortalidade.

\begin{abstract}
Objective: To conduct a literature review to determine the effects of comorbidities on the recovery of the elderly after hip fracture surgery and the risks to mortality of this patient. Methods: This is an integrative review of a qualitative approach. Performed in the following databases LILACS, MEDLINE, SCIELO and PUBMED, using the following Health Sciences Descriptors (decs): Falls; Elderly; Hip fractures; Comorbiditie and Mortality. Results: In the analysis of the studies it was found that the highest frequency of falls and hip fractures is among the elderly women, and the postoperative period is related to the emergence of comorbidities related to physical function deficit, body pain and fear. Of new falls. Patients who remain hospitalized longer after surgery have a greater chance of comorbidities, compromising their recovery, thus providing higher risks to premature mortality of the elderly. Final Considerations: Patients with hip fractures who have postoperative comorbidities are predisposed to clinical complications and have a high mortality rate. In this sense, there is a
\end{abstract}

\footnotetext{
${ }^{1}$ Mestrando da Atenas College University, Orlando-Flórida-USA. *E-mail: allexsobralfisio@hotmail.com

${ }^{2}$ Faculdade do Juazeiro do Norte (FJN), Juazeiro do Norte-Ceará.
} 
need to develop actions that guarantee the protection and recovery of this elderly, in order to reduce the risks to mortality after hip fracture.

Keywords: Falls, Elderly, Hip fractures, Comorbiditie, Mortality.

\section{RESUMEN}

Objetivo: Realizar una revisión de la literatura para determinar los efectos de las comorbilidades en la recuperación de los ancianos después de una cirugía de fractura de cadera y los riesgos de mortalidad de este paciente. Métodos: Esta es una revisión integradora de un enfoque cualitativo. Realizado en las siguientes bases de datos LILACS, MEDLINE, SCIELO y PUBMED, utilizando los siguientes descriptores de ciencias de la salud (decs): Caídas; Ancianos; Fracturas de cadera; Comorbilidade y Mortalidad. Resultados: En el análisis de los estudios se encontró que la frecuencia más alta de caídas y fracturas de cadera se encuentra entre las mujeres de edad avanzada, y el período postoperatorio está relacionado con la aparición de comorbilidades relacionadas con el déficit en las funciones físicas, el dolor corporal y el miedo de nuevas caídas. Los pacientes que permanecen hospitalizados por más tiempo después de la cirugía tienen una mayor probabilidad de comorbilidades, lo que compromete su recuperación y, por lo tanto, proporciona mayores riesgos de mortalidad prematura de los ancianos. Consideraciones finales: Los pacientes con fracturas de cadera que tienen comorbilidades postoperatorias están predispuestos a complicaciones clínicas y tienen una alta tasa de mortalidad. En este sentido, existe la necesidad de desarrollar acciones que garanticen la protección y recuperación de este anciano, a fin de reducir los riesgos de mortalidad después de una fractura de cadera.

Palabras clave: Caídas, Ancianos, Fracturas de cadera, Comorbilidade, Mortalidad.

\section{INTRODUÇÃO}

Nas últimas décadas no Brasil verificou-se um crescente aumento da população idosa, isso acontece devido ao aumento da expectativa de vida desta população. De acordo com o Instituto Brasileiro de Geografia e Estatística (IBGE), a população de idosos no Brasil hoje já ultrapassa o número de 32 milhões de pessoas acima dos 60 anos e as estimativas para os próximos vinte e cinco anos indicam que poderá exceder os sessenta e cinco milhões de idosos no fim desse período, quando representará cerca $31 \%$ da população brasileira (IBGE, 2016).

Neste contexto do envelhecimento populacional, observa-se os problemas decorrentes deste público, dentre eles destaca-se as fraturas em idosos e seus impactos. As fraturas em idosos são um problema de saúde pública, estima-se que, apenas os acidentes ocasionados por quedas em idosos que resultam em fraturas, seja responsável por cerca de 10 mil mortes por ano em todo Brasil. Essa situação é pior nos centros urbanos, principalmente nas capitais e regiões metropolitanas, onde é crescente o número de intercorrências por quedas com desfecho de fratura em idoso (VERAS RP e OLIVEIRA M, 2018).

Aproximadamente $11,4 \%$ dos episódios de queda desencadeiam fraturas, sendo as mais comuns são as umerais, vertebral, no fêmur, rádio distal e quadril. As fraturas de quadril são ocorrências muito comuns e sérias em pacientes idosos, representa um grande problema de saúde pública devido ao seu impacto substancial na assistência médica e nos custos com saúde. Atualmente 135 mil fraturas de quadril por ano no Brasil, estimativas revelam que aproximadamente 4,7 milhões de fraturas de quadril ocorrerão em todo 0 Brasil até 2050 (CABERLON IC e BOS AJG, 2015).

No ponto de vista da saúde do idoso, há uma atenção crescente relacionada aos impactos das fraturas quadril e às morbidades associadas, em decorrência do aumento dos custos com os cuidados à saúde bem como a melhora e recuperação destes idosos. Os determinantes para a recuperação deste idoso fundamentase em quatro princípios básicos: funcionalidade, independência, bem-estar, alterações do humor, podem estas bases serem afetadas por diferentes fatores frente a recuperação deste paciente e, como consequência, influenciar o tratamento, que deve ser individualizado. 
A medida que a atenção após fratura de quadril é importante para a avaliação dos efeitos do tratamento e do atendimento pelos serviços de saúde no bem-estar dos idosos. As fraturas de quadril demanda custos a saúde, entender os tipos de impacto que as intervenções de cuidados à saúde têm na vida deste paciente e o custo-efetividade do tratamento após fratura de quadril no idoso (NASCIMENTO JS e TAVARES DMS, 2016).

Estima-se que uma em cada quatro mulheres e um em cada doze homens irão sofrer esse tipo de fratura, sendo a maioria das fraturas de quadril ( $80 \%$ ) ocorrem em pessoas com 60 anos ou mais. Dentre os principais fatores de risco que predispõem as quedas e, consequentemente, fraturas de quadril, estão, osteoporose, demências, acuidade visual diminuída, déficits sensoriais, acidente vascular cerebral (AVC), hipotrofia muscular, fraqueza muscular, alteração de equilíbrio e dos reflexos, deformidades osteomioarticulares, doenças neurológicas e cardiovasculares (RIZK P, et al., 2016).

Na sua grande maioria após a fratura de quadril uma intervenção cirúrgica é indicada. O tratamento conservador só é adotado quando não se observa desvio e em casos que a fratura é incompleta. $O$ procedimento cirúrgico para o tratamento da fratura de quadril no idoso quase sempre é complicado, reflete a importância de um acompanhamento clínico desses pacientes, bem como a avaliação adequada de seu risco cirúrgico.

Diversos estudos apontam a idade avançada, cor branca, estado físico, período de internação, presença de demência, sexo feminino, comorbidades e o atraso do tratamento, como fatores para os altos índices de mortalidade pós-cirúrgicas (FREITAS MG, et al., 2015).

As comorbidades pós-cirurgia reparadora da fratura de quadril no idoso apresenta efeitos prejudiciais sobre o sistema musculoesquelético, pode-se citar a incapacidade de locomoção, atrofia muscular, fragilidade óssea, imobilidade, novas quedas e fraturas, degeneração articular e a intensificação da osteoporose.

O comprometimento funcional ao idoso com fraturas de quadril, aliada as comorbidades significativas e $o$ alto risco de complicações pós-cirúrgicas, essas condições somadas apresentam taxas de mortalidade elevadas quando comparadas com outros tipos de fraturas (MATIAS AGC, et al., 2015).

Assim, compreendendo a importância do episódio de quedas para os idosos, a elevada incidência de fraturas de quadril nesta população, a importância do planejamento de ações voltadas a minimiza as comorbidades e seus riscos a mortalidade pós-cirurgia de fratura de quadril em idosos.

Neste contexto, este estudo se propõe a avaliar o conhecimento científico produzido, relacionado às quedas e fraturas de quadril em idosos, suas comorbidades e os riscos a mortalidade pós-cirúrgica. Diante do contexto surgiu o seguinte questionamento: Quais são os impactos das comorbidades na recuperação dos idosos com fratura de quadril e os riscos para sua mortalidade pós-cirúrgica?

Este estudo tornou-se relevante pois, possibilita a reunião de informações acerca das quedas e fratura de quadril na população idosa, analisar os efeitos das comorbidades na recuperação do idoso e os riscos associados à mortalidade pós-cirurgia desses pacientes. Objetivou-se realizar um levantamento na literatura para determina os efeitos das comorbidades na recuperação dos idosos pós-cirurgia de fratura de quadril e os riscos à mortalidade deste paciente.

\section{MÉTODOS}

O presente estudo define-se como uma revisão integrativa da literatura com abordagem qualitativa. A abordagem qualitativa refere-se a um método de pesquisa e investigação científica que se foca no caráter subjetivo do objeto analisado, estudando as suas particularidades e experiências individuais (TAQUETTE SR e MINAYO MC, 2016).

Neste sentido, buscou-se publicações científicas brasileira, indexadas nas seguintes bases de dados: Literatura Latino-Americana e do Caribe em Ciências da Saúde (LILACS), Medical Literature Analysis and Retrieval System Online (Medline), Scientific Electronic Library Online (scielo) e US National Library of Medicine (pubmed). Utilizando-se da combinação dos seguintes Descritores em Ciências da Saúde (decs) na pesquisa: quedas, idosos, fraturas do quadril, comorbidade e mortalidade. A pesquisa foi realizada nos meses de outubro e novembro de 2019 , concomitantemente nas quatro bases de dados. 
Para busca da literatura adotaram-se como critérios de inclusão artigos inteiramente disponíveis relacionados ao objeto da pesquisa, nos idiomas português e inglês, originados de periódicos nacionais e internacionais, sendo citados no mínimo dois descritores utilizados no estudo e indexados nas bases de dados referidas com data de publicação dos últimos 5 anos, e como critérios de exclusão optou-se por não artigos que não tivessem disponíveis na forma gratuita, textos incompletos e que não estivessem disponíveis na

As informações científicas foram detalhadas e divididas observando: título do trabalho, autores, ano da publicação, revista científica, objetivo do estudo e resultados. A parte dos estudos encontrados relacionados aos descritores e que atenderam os critérios de inclusão e expulsão, obteve-se 38 artigos científicos que após leitura e análise criteriosa foram utilizados 10 artigos com o intuito de abranger o conhecimento produzido sobre o tema discutido na revisão interativa.

Figura 1 - Fluxograma mostrando a seleção do estudo para revisão. Juazeiro do Norte (CE), Brasil, 2019.
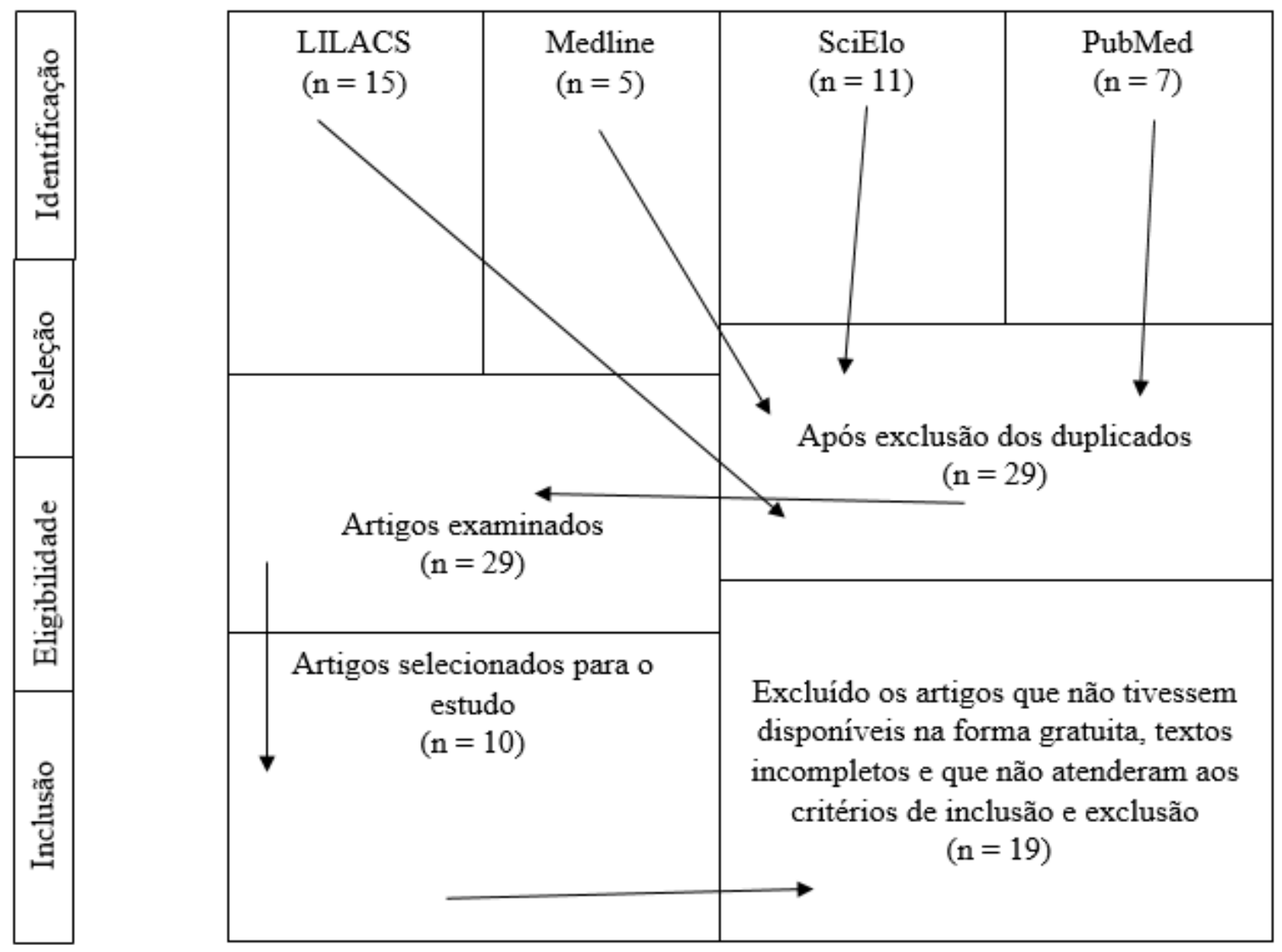

Fonte: Sousa AAS, et al., 2019.

\section{RESULTADOS}

A pesquisa na literatura resultou em dez artigos (Figura 1) nos quais tratavam das consequências das comorbidades na recuperação dos idosos pós-cirurgia de fratura de quadril e os riscos à mortalidade deste paciente, onde as informações retiradas estão expostas no (Quadro 1).

De acordo com o vínculo da instituição de origem dos autores principais, nove artigos estão vinculados a universidades e um está vinculado a uma Fundação. Em relação à caracterização dos estudos, quanto ao ano de publicação, em 2015 foram encontrados 3 artigos acerta do tema em discussão, nos anos de 2018 e 2017, foram publicados dois artigos em cada ano e nos anos de 2019 e 2016 um artigo cada. No que se refere ao idioma, oito publicações foram em português e duas em inglês. Quanto ao periódico de publicação, foram detectados quatro periódicos diferentes, sendo sete revistas específicas de Ortopedia. 
Quadro 1 - Dados de título do trabalho, autores, ano da publicação, revista científica, objetivo do estudo e resultados. Juazeiro do Norte (CE), Brasil, 2019.

\begin{tabular}{|c|c|c|c|c|c|}
\hline & Título do trabalho & $\begin{array}{l}\text { Autores/an } \\
\text { o da } \\
\text { publicação }\end{array}$ & $\begin{array}{c}\text { Revista } \\
\text { científic } \\
\text { a }\end{array}$ & $\begin{array}{l}\text { Objetivo do } \\
\text { estudo }\end{array}$ & Resultados \\
\hline 1 & $\begin{array}{l}\text { Custo-efetividade do } \\
\text { tratamento cirúrgico } \\
\text { das fraturas de } \\
\text { quadril em idosos no } \\
\text { Brasil }\end{array}$ & $\begin{array}{l}\text { Loures FB, } \\
\text { et al., } 2015 .\end{array}$ & $\begin{array}{l}\text { Revista } \\
\text { Brasileir } \\
\text { a de } \\
\text { Ortopedi } \\
\quad \text { a }\end{array}$ & $\begin{array}{l}\text { Estimar o custo por ano de vida } \\
\text { ajustado pela qualidade (QALY), com } \\
\text { foco no tempo entre o trauma e a } \\
\text { cirurgia. }\end{array}$ & $\begin{array}{c}\text { As variáveis de confusão idade, sexo, risco anestésico (ASA), tipo } \\
\text { de fratura e tipo de cirurgia foram controladas para. As amostras } \\
\text { mostraram-se homogêneas em relação a essas variáveis. O } \\
\text { período de internação e alta hospital se mostra variáveis }(3-27, \\
\text { dias), observou-se que a maior permanência aumentava os riscos } \\
\text { de mortalidade. }\end{array}$ \\
\hline 2 & $\begin{array}{l}\text { Avaliação da } \\
\text { qualidade de vida } \\
\text { submetidos à } \\
\text { hemiartroplastia dos } \\
\text { pacientes do quadril }\end{array}$ & $\begin{array}{l}\text { Abreu EL, } \\
\text { Oliveira } \\
\text { MHA, } 2015 .\end{array}$ & $\begin{array}{l}\text { Revista } \\
\text { Brasileir } \\
\text { a de } \\
\text { Ortopedi } \\
\quad \text { a }\end{array}$ & $\begin{array}{l}\text { Análise comparativa da qualidade de } \\
\text { vida dos pacientes submetidos à } \\
\text { hemiartroplastia do quadril, no pré- } \\
\text { operatório, com três e seis meses de } \\
\text { operados, por meio do questionário } \\
\text { SF3. }\end{array}$ & $\begin{array}{c}\text { Com relação à saúde física, os pacientes apresentaram baixa na } \\
\text { capacidade funcional e nos aspectos físicos. Demostrando } \\
\text { comorbidades do sistema muscular, fragilidade óssea e } \\
\text { degeneração articula. }\end{array}$ \\
\hline 3 & $\begin{array}{l}\text { Análise econômica } \\
\text { do tratamento } \\
\text { cirúrgico de fratura } \\
\text { do quadril em idosos }\end{array}$ & $\begin{array}{l}\text { Loures FB, } \\
\text { et al., } 2015 .\end{array}$ & $\begin{array}{l}\text { Revista } \\
\text { de } \\
\text { Saúde } \\
\text { Pública }\end{array}$ & $\begin{array}{l}\text { Analisar a razão custo-utilidade } \\
\text { incremental do tratamento cirúrgico da } \\
\text { fratura do quadril de pacientes idosos. }\end{array}$ & $\begin{array}{c}\text { O custo direto médico mostrou-se aumentado de forma } \\
\text { estatisticamente significativa entre os pacientes da alternativa } \\
\text { tardia. A mortalidade intra-hospitalar foi maior no grupo da } \\
\text { cirurgia tardia ( } 7,4 \% \text { contra } 16,9 \%) \text {. }\end{array}$ \\
\hline 4 & $\begin{array}{l}\text { Complicações da } \\
\text { artroplastia total do } \\
\text { quadril em pacientes } \\
\text { com e sem diabetes } \\
\text { mellitus controlada } \\
\text { durante a internação }\end{array}$ & $\begin{array}{l}\text { Falcão } \\
\text { FRC, et al., } \\
\quad 2016 .\end{array}$ & $\begin{array}{l}\text { Revista } \\
\text { Brasileir } \\
\text { a de } \\
\text { Ortopedi } \\
\quad \text { a }\end{array}$ & $\begin{array}{l}\text { Avaliar a associação entre } \\
\text { complicações da ATQ e DM } \\
\text { controlada durante o período de } \\
\text { internação. }\end{array}$ & $\begin{array}{l}\text { A maioria dos pacientes eram mulheres idosas. As comorbidades } \\
\text { mais prevalentes no período de internação foram osteoporose, } \\
\text { dermatite das fraldas e infeção do trato urinário. }\end{array}$ \\
\hline
\end{tabular}

REAS/EJCH | Vol.Sup.n.41 | e2939 | DOI: https://doi.org/10.25248/reas.e2939.2020 Página 5 de 12 


\section{Revista Eletrônica Acervo Saúde / Electronic Journal Collection Health ｜ISSN 2178-2091}

\begin{tabular}{|c|c|c|c|c|c|}
\hline 5 & $\begin{array}{l}\text { Avaliação da } \\
\text { efetividade de um } \\
\text { programa de atenção } \\
\text { ao idoso com fratura } \\
\text { de quadril: uma } \\
\text { estratégia de rede }\end{array}$ & $\begin{array}{l}\text { Farias FID, } \\
\text { et al., } 2017 .\end{array}$ & $\begin{array}{l}\text { Revista } \\
\text { Brasileir } \\
\text { a de } \\
\text { Geriatria } \\
\text { e } \\
\text { Gerontol } \\
\text { ogia }\end{array}$ & $\begin{array}{l}\text { Avaliar a efetividade de um programa } \\
\text { de atenção ao idoso com fratura de } \\
\text { quadril decorrente de queda, aplicado } \\
\text { a uma rede pública de assistência, no } \\
\text { município de } \\
\text { Canoas, no Rio Grande do Sul, Brasil. }\end{array}$ & $\begin{array}{l}\text { A morbidade mais frequente foi a hipertensão. Após a } \\
\text { implementação do programa houve redução do tempo médio } \\
\text { decorrido entre a fratura e o início da cirurgia, da média de } \\
\text { permanência, da taxa de infecção do trato urinário, da taxa de } \\
\text { óbito e dos custos assistenciais. }\end{array}$ \\
\hline 6 & $\begin{array}{l}\text { Mortalidade em um } \\
\text { ano de pacientes } \\
\text { idosos com fratura } \\
\text { do quadril tratados } \\
\text { cirurgicamente num } \\
\text { hospital do Sul do } \\
\text { Brasil }\end{array}$ & $\begin{array}{c}\text { Guerra } \\
\text { MTE, et al., } \\
2017 .\end{array}$ & $\begin{array}{l}\text { Revista } \\
\text { Brasileir } \\
\text { a de } \\
\text { Ortopedi } \\
\quad \text { a }\end{array}$ & $\begin{array}{l}\text { Analisar a mortalidade, em um ano de } \\
\text { seguimento, de pacientes com fratura } \\
\text { da extremidade proximal do fêmur } \\
\text { submetidos a procedimento cirúrgico } \\
\text { no hospital universitário da nossa } \\
\text { instituição. }\end{array}$ & $\begin{array}{c}\text { A taxa de mortalidade em um ano foi de } 23,6 \% \text {. A mortalidade foi } \\
\text { maior em mulheres, numa proporção } 3: 1 \text {. Pacientes que } \\
\text { permaneceram internados por até } 15 \text { dias e os que tiveram alta } \\
\text { hospitalar em até sete dias após a cirurgia apresentaram um } \\
\text { aumento na sobrevida. }\end{array}$ \\
\hline 7 & $\begin{array}{l}\text { Tratamento de } \\
\text { fraturas } \\
\text { intertrocanterianas } \\
\text { estáveis do fêmur } \\
\text { com haste femoral } \\
\text { proximal versus } \\
\text { parafuso dinâmico } \\
\text { do quadril: estudo } \\
\text { comparativo }\end{array}$ & $\begin{array}{l}\text { Sharma A, } \\
\text { et al., } 2018 .\end{array}$ & $\begin{array}{l}\text { Revista } \\
\text { Brasileir } \\
\text { a de } \\
\text { Ortopedi } \\
\quad \text { a }\end{array}$ & $\begin{array}{l}\text { Avaliar e comparar os resultados } \\
\text { clínicos e radiológicos de pacientes } \\
\text { com fraturas intertrocantéricas } \\
\text { estáveis tratados com unha femoral } \\
\text { proximal versus parafuso dinâmico do } \\
\text { quadril. }\end{array}$ & $\begin{array}{l}\text { Nos acompanhamentos mensais de três e seis meses, o grupo do } \\
\text { parafuso dinâmico do quadril apresentou aparecimento de } \\
\text { comorbidades devido sua recuperação tardia, tais como, úlceras } \\
\text { de decúbito, rigidez articular e declínio da massa óssea. }\end{array}$ \\
\hline
\end{tabular}

REAS/EJCH | Vol.Sup.n.41 | e2939 | DOI: https://doi.org/10.25248/reas.e2939.2020 Página 6 de 12 


\begin{tabular}{|c|c|c|c|c|c|}
\hline 8 & $\begin{array}{l}\text { Internação e } \\
\text { mortalidade por } \\
\text { quedas em idosos no } \\
\text { Brasil: análise de } \\
\text { tendência }\end{array}$ & $\begin{array}{l}\text { Abreu } \\
\text { DROM, et } \\
\text { al., } 2018 \text {. }\end{array}$ & $\begin{array}{l}\text { Ciência } \\
\text { \& Saúde } \\
\text { Coletiva }\end{array}$ & $\begin{array}{l}\text { O objetivo deste artigo é analisar a } \\
\text { tendência da morbimortalidade por } \\
\text { quedas em idosos, no Brasil, no } \\
\text { período de } 1996 \text { a } 2012 \text {. }\end{array}$ & $\begin{array}{l}\text { As taxas de mortalidade aumentaram em todas regiões e nas } \\
\text { capitais dos estados, para as internações houve variação da } \\
\text { tendência das taxas nas regiões e nas capitais do país. As taxas } \\
\text { variaram de } 2,58 \text { a } 41,37 \text { internações por } 10.000 \text { idosos em } 1996 \\
\text { e } 2012 \text {, respectivamente, e as taxas de mortalidade aumentaram } \\
\text { de } 1,25 \text {, em } 41996 \text {, a } 3,75 \text {, em } 2012 \text {, por } 10.000 \text { idosos. }\end{array}$ \\
\hline 9 & $\begin{array}{l}\text { Comorbidades, } \\
\text { intercorrências } \\
\text { clínicas e fatores } \\
\text { associados à } \\
\text { mortalidade em } \\
\text { pacientes idosos } \\
\text { internados por } \\
\text { fratura de quadril }\end{array}$ & $\begin{array}{l}\text { Edelmuth } \\
\text { SVCL, et } \\
\text { al., } 2018 .\end{array}$ & $\begin{array}{l}\text { Revista } \\
\text { Brasileir } \\
\text { a de } \\
\text { Ortopedi } \\
\quad \text { a }\end{array}$ & $\begin{array}{l}\text { Analisar as comorbidades e as } \\
\text { intercorrências clínicas e determinar } \\
\text { os fatores associados à mortalidade } \\
\text { de pacientes idosos internados por } \\
\text { fratura de quadril em um hospital } \\
\text { público de atenção terciária. }\end{array}$ & $\begin{array}{c}\text { A maioria dos pacientes }(50,7 \%) \text { tinha duas ou mais } \\
\text { comorbidades. A taxa de mortalidade durante a internação foi de } \\
11,9 \% \text { e esteve diretamente vinculada à presença de infecções } \\
\text { no período hospitalar. }\end{array}$ \\
\hline $\begin{array}{l}1 \\
0\end{array}$ & $\begin{array}{c}\text { Maior mortalidade } \\
\text { em } 30 \text { dias } \\
\text { associada ao uso de } \\
\text { hastes } \\
\text { intramedulares em } \\
\text { comparação com } \\
\text { parafusos } \\
\text { deslizantes de } \\
\text { quadril para o } \\
\text { tratamento de } \\
\text { fraturas de quadril } \\
\text { trocantéricas. }\end{array}$ & $\begin{array}{c}\text { Whitehouse } \\
\text { MR, et al., } \\
2019 .\end{array}$ & $\begin{array}{l}\text { Revista } \\
\text { Brasileir } \\
\text { a de } \\
\text { Ortopedi } \\
\quad \text { a }\end{array}$ & $\begin{array}{l}\text { O objetivo deste estudo foi investigar a } \\
\text { associação entre o tipo de operação } \\
\text { utilizada para tratar uma fratura } \\
\text { trocantérica do quadril e a mortalidade } \\
\text { em } 30 \text { dias. }\end{array}$ & $\begin{array}{l}\text { O uso de hastes intramedulares curtas e longas foi associado a } \\
\text { um aumento na mortalidade em comparação com o uso de } \\
\text { parafusos deslizantes. Isso se deu pela maior permanência } \\
\text { internado pós-cirurgia dos pacientes com hastes intramedulares. }\end{array}$ \\
\hline
\end{tabular}

Fonte: Sousa AAS, et al., 2019. 
A análise dos artigos, permitiu a identificação temática de três categorias: avaliação e tratamento dos idosos com fratura de quadril (cinco artigos), complicações decorrentes do trauma (um artigo) e mortalidade pós-cirúrgica de fratura de quadril (quatro artigos). Onde estão demonstradas no (Tabela 1).

Tabela 1 - Categorias temáticas dos estudos de revisão integrativa. Juazeiro do Norte (CE), Brasil, 2019.

\begin{tabular}{lcc}
\hline & Categorias & Artigos \\
\hline $\mathbf{1}$ & Avaliação e tratamento dos idosos com fratura de quadril & $1,2,3,5$ e 7 \\
2 & Complicações decorrentes do trauma & 4 \\
3 & Mortalidade pós-cirúrgica de fratura de quadril & $6,8,9$ e 10 \\
\hline
\end{tabular}

Fonte: Sousa AAS, et al., 2019.

\section{DISCUSSÃO}

\section{Categoria 1: Avaliação e tratamento dos idosos com fratura de quadril}

O envelhecimento da população idosa no Brasil tem resultado em um aumento proporcional no número de idosos com incapacidade crônica durante essa fase da vida, as quedas que resultam em fraturas são um grave problema de saúde pública entre os idosos por causa de sua frequência. Dentre os tipos de fraturas destaca-se a de quadril devido seu grande impacto sobre a vida do idoso, a fratura de quadril é um acontecimento que demanda um elevado custo ao Sistema Único de Saúde (SUS), por consequência da alta complexibilidade na avaliação do paciente, do tratamento cirúrgico e pelo período de internação hospitalar do idoso (CARNEIRO JA, et al., 2016).

Os idosos mais longevos e que sofreram fraturas de quadril apresentaram um maior período de internação hospitalar que os demais como menor faixa etária (BRIGGS R, et al., 2018). Assim, surgem relação quando outros autores Barros IFO, et al. (2015), afirmam que idosos com maior faixa etária apresentam menor capacidade para atividades de vida diária (avds) após fratura quadril, onde demonstra existe um declínio nas capacidades físicas resultando em idosos menos independentes, desenvolvendo fragilidades e instabilidades, tais fatores contribuem para perda da autonomia do idoso e dificulta a recuperação precoce deste grupo.

Diversos estudos associam fatores econômicos e tratamento adequado como sendo aspectos essenciais a melhor recuperação das fraturas de quadril em idosos. Em estudo realizado por Stolnicki B e Oliveira LG (2016), com 316 pacientes que foram atendidos e tratados preventivamente com FFP, tiveram um índice de alta hospitalar precoce $(26,6 \%)$ e redução da mortalidade intra-hospitalar $(17,8 \%)$. Esses dados colaboram com a pesquisa bibliográfica onde determina que o tratamento preventivo pós-operatório apresentou diminuição da taxa de $23,15 \%$ de mortalidade no primeiro ano entre os idosos com fratura de fêmur (OLIVEIRA TC, et al., 2015).

Neste contexto, percebe-se a necessidade de avaliar a efetividade do procedimento cirúrgico de fratura de quadril nos idosos e sua importância na relação de: observar os resultados da intervenção cirúrgica, levantar questionamento sobre a melhor conduta pós-cirúrgica e realizar avaliação da relação custo-benefício das intervenções médicas. Sendo assim, foi verificado que o tempo permanecido hospitalizado pelo idoso desde o momento da admissão, o procedimento cirúrgico e alta hospitalar pós-cirurgia são eventos sinais na busca da recuperação deste paciente (GOVEIA VR, et al., 2015).

Estudo desenvolvido com 267 idoso com fratura de quadril o tempo praticado da admissão hospitalar até o momento da cirurgia foi 9,6 dias, com permanência média de 14,2 dias. O tempo até a cirurgia influenciou nos resultados óbito intra-hospitalar de forma clinicamente significativa, onde a maior permanência hostilizado do idoso está relacionada diretamente com surgimento de complicações, levando tais pacientes ao declínio funcionar, morbidade e mortalidade (VICENTE JRM, et al., 2015).

O tempo ideal entre a fratura de quadril e a instituição do tratamento cirúrgico tem sido amplamente debatido na literatura. $O$ período ideal para cirurgia está estabelecido entre 24 a 48 horas após a fratura. 0 tempo de intenção hospitalar no pós-cirúrgico do idoso mostrou-se significativo para sua recuperação, onde, 
pacientes que permaneceram internados por mais de 30 dias apresentaram aparecimentos de comorbidades, como, úlcera de decúbito, dermatite das fraldas, escabiose e micoses superficiais, bem como, aumento na mortalidade (GOPINATH B, et al., 2016).

\section{Categoria 2: Complicações decorrentes do trauma}

As quedas são eventos sentinelas da saúde da pessoa idosa, a ocorrência de uma queda pode desencadear a fratura, uma fratura pode gerar consequências negativas nas condições saúde do idoso. Onde, a maior prevalência de quedas entre os idosos são aqueles mais longevos (entre a sétima e oitava década de vida), porque as alterações musculoesqueléticas podem comprometer o desempenho de habilidades motoras, comprometimento da marcha, equilíbrio, dificultar a adaptação do indivíduo ao ambiente, força muscula diminuída e predispô-lo às quedas (LITWIN H, et al., 2018).

Além da marcha e equilíbrio que são fatores do sistema musculoesquelético, também foram identificadas outras consequências de características clinicas, como arritmia e insuficiência cardíaca, problemas respiratórios, bem como acidente vascular encefálico e depressão no ambiente hospitalar.

Estudo epidemiológico realizado com idosos após a ocorrência fratura de quadril em hospital-escola da região central do Rio de Janeiro demonstrou que, dos 167 idosos que sofreram quedas, 39 tiveram fratura de quadril. Apresentando fratura associadas às doenças crônicas (especialmente insuficiência cardíaca) e contribui para uma taxa de $8,2 \%$ de mortalidade pós-operatória antes da alta hospitalar (KERNKAMP CL, et al., 2016).

Destaca-se, ainda, que à fratura de quadril em idosos é resultado de um trauma de baixo impacto, na maioria das vezes decorrente de quedas, as quais são cada vez mais prováveis em pessoas com 60 anos ou mais. Estudos demonstraram que as mulheres estão mais suscetíveis às quedas com desfecho de fratura de quadril, ressaltando, também, o risco da prevalentes de óbito e complicações em mulheres idosas pós-cirurgia de fratura de quadril decorrentes das complicações do trauma e das comorbidades (RODRIGUES GD, et al., 2016).

Neste sentido, observa-se a maior prevalência de fratura de quadril em mulheres estar relacionada ao fato do sexo feminino ter maior expectativa de vida e, portanto, iniciar o processo de declínio de massa óssea antes do homem, estar mais exposto a fatores de risco, ter maior suscetibilidade a quedas, além das influências particulares como peso/altura e fatores genéticos relacionados ao sexo feminino (BRETANHA AF, et al., 2015).

O risco da ocorrência de fratura quadril aumenta significativamente com o avançar da idade em mulheres com mais de 70 anos. Este senário se torna mais preocupante entre as mulheres idosas que vivem em lares de longa permanência e que sofram fratura de quadril, onde o aparecimento de complicações pós cirurgia reparadora de fratura de quadril teve um aumento em $30 \%$ da possibilidade da presença de comorbidades durante a recuperação destas idosas que se encontram institucionalizadas.

Após fratura quadril, cerca de $12 \%$ dos idosos voltam a ser hospitalizados devido a ocorrência de agravos a saúde e dificuldade na sua recuperação, principalmente relacionados a problemas renais, respiratórios e mediante o aparecimento de comorbidades (PIMENTEL WRT, et al., 2015).

Um estudo prospectivo com 329 pacientes idosos com fratura de quadril e submetidos a cirurgia a mais de 15 dias de internação, apresentava, 37,3\% tinham uma comorbidade, 17,9\% tinham duas comorbidades, $22,3 \%$ apresentaram três condições a comorbidades e 10,4\% da população estudada foram verificadas mais de quatro comorbidades, destacando assim, que surgimento de comorbidades no paciente idoso pós-cirurgia de fratura de quadril é determinante para um desfecho desfavorável a sua recuperação (PRATO SCF, et al., 2017).

\section{Categoria 3: Mortalidade pós-cirúrgica de fratura de quadril}

Pacientes idosos com fratura de quadril tem maior risco para mortalidade, aumentando a necessidade de cuidados e supervisão, ficam impossibilitados de retornar às condições de vida anteriores, aumentam as chances de fraturas secundárias, diminuem a capacidade de mobilidade e locomoção. Sabe-se que os idosos que permanecem hospitalizados por longo período apresentam características, como diminuição da 
capacidade funcional e decréscimo da aptidão física, que, entre outros fatores, contribuem para perda da autonomia que está racionada com o aumento das comorbidades (TYROVOLAS S, et al., 2016). Em relevância a mortalidade dos idosos após queda e fratura, foi comprovado que os idosos com maior medo de uma fratura posterior foram os que mais as sofreram e apresentaram piores resultados para capacidade funcional e perda da autonomia. Idosos que sofreram fraturas relataram mais medo de sofrê-las do que os que nunca tiveram fratura.

Entre as mulheres na faixa etária de 60 a 69 anos, apresentam maior medo de sofrer uma fratura, em relação aquelas que apresentaram de moderado a alto medo da ocorrência de fratura revelaram piores índices para funções física, social, funcional, dor corporal, vitalidade e saúde geral, e os homens com moderado grau de medo de sofrerem fraturas apresentaram piores dados para as funções social e funcional e para saúde geral.

Estes fatores são negativos para a recuperação do idoso, possibilitando assim, aumentando os riscos a mortalidade precoce deste paciente e favorecendo o senário para o surgimento de comorbidades nos idosos pós fratura de quadril (MENTIS M, et al., 2017).

Muitos idosos com histórico de fratura de quadril apresentam maiores chances de sofrer novas quedas e possivelmente uma nova fratura. Estas em sua maioria, podem ser prevenidas e evitadas, diminuindo as consequências, como o aparecimento de comorbidades nos idosos, onde elas podem gerar redução ou perda da autonomia, da qualidade de vida, da independência, aumentando do gasto financeiros e dos danos sociais, mentais e emocionais, levando à institucionalização, hospitalização e aumento da morbidade e mortalidade (OLIVEIRA CC e BORBA VZC, 2017).

O número de comorbidades pós-cirúrgicas influencia na mortalidade dos idosos com fraturas de quadril e que a presença de duas ou mais comorbidades está associada a um aumento (21\%) da taxa de mortalidade nesta população. A hostilização prolongada do idoso pode favorecer um aumento de mais de quatro vezes na mortalidade em pacientes com mais de 10 dias de internação hospitalar no pós-cirurgia de fratura de quadril em comparação com aqueles que permaneceram menos de 10 dias (FUHRMANN AC, et al., 2015).

O tempo hospitalizado pelo idoso desde o momento da fratura de quadril até o instante do procedimento cirúrgico é determinante para uma boa recuperação do idoso. A demora no atendimento pode acarretar pioria dos resultados clínicos e de qualidade de vida deste paciente, além de aumentar os gastos em saúde, com maior permanência do idoso no centro hospitalar e custos com profissionais e materiais. $O$ tempo dispendido entre o momento da internação hospitalar e a cirurgia é devido à demora no preparo do paciente préoperatório e disponibilidade de salas e materiais necessários à cirurgia, o tempo até a cirurgia influenciou diretamente nos custos-efetividade do tratamento, com o aumento da permanência internado pelo idoso favorece o aparecimento de comorbidades e aumenta os ricos a mortalidade (CALHA A, 2015).

A mortalidade pós-fratura de quadril no idoso permanece elevada não somente nos meses subsequentes ao evento, mas por anos depois do trauma. Sua lenta recuperação pós-cirúrgica, somada ao aparecimento de comorbidades e a idade elevada deste paciente, sendo assim, um senário propicio ao declínio da saúde do idoso favorecendo o aumento da sua mortalidade. As fraturas quadril no idoso constituem as fraturas mais frequentemente operadas e com maior taxa de mortalidade pós-cirúrgica, tornaram-se uma questão de saúde pública, devido ao alto custo do tratamento e à dificuldade de recuperação e independência funcional deste paciente (SMITH AA, et al., 2017).

\section{CONSIDERAÇÕES FINAIS}

Neste estudo foi possível identificar as principais comorbidades que acometer o idoso pós-cirurgia de fratura de quadril, a prevenção dos fatores de risco são medidas importantes na melhoria do prognóstico a curto prazo desses indivíduos. Apesar das consequências das comorbidades serem bastante prejudicais ao idoso, medidas que diminua o tempo de permanência internado, redução das complicações pós-cirúrgica e tratamento adequado após alta hospitalar, são meios que garante a proteção e a recuperação deste paciente reduzindo seus riscos a morbidade e mortalidade. Assim, Ações que promova a recuperação precoce deste idoso devem ser estabelecidas para evitar aparecimento de doenças adicionais, sendo este o posto principal e desta forma diminuir os índices de mortalidade pós-cirurgia de fratura de quadril.

REAS/EJCH | Vol.Sup.n.41 | e2939 | DOI: https://doi.org/10.25248/reas.e2939.2020 Página 10 de 12 


\section{REFERÊNCIAS}

1. ABREU DROM, et al. Internação e mortalidade por quedas em idosos no Brasil: análise de tendência. Ciência da Saúde Coletiva, Rio de Janeiro, 2018; 23(4): 1131-1141.

2. ABREU EL, OLIVEIRA MHA. Avaliação da qualidade de vida submetidos à hemiartroplastia dos pacientes do quadril. Revista Brasileira de Ortopedia, São Paulo, 2015; 50(5): 530-536.

3. BARROS IFO, et al. Internações hospitalares por quedas em idosos brasileiros e os custos correspondentes no âmbito do sistema único de saúde. Revista Kairós, 2015; 18(4): 63-80.

4. BRETANHA AF, et al. Sintomas depressivos em idosos residentes em áreas de abrangência das unidades básicas de saúde da zona urbana de Bagé, RS. Revista Brasileira de Epidemiologia, 2015; 18(1): 1-12.

5. BRIGGS R, et al. Does baseline depression increase the risk of unexplained and accidental falls in a cohort of community dwelling older people? Data from the irish longitudinal study on ageing (TILDA). International Jounal of Geriatric Psychiatry, 2018; 33(2): 205-211.

6. CABERLON IC, BOS AJG. Diferenças sazonais de quedas e fraturas em idosos gaúchos. Ciência Saúde Coletiva, Rio de Janeiro, 2015; 20(12): 3743-3752.

7. CALHA A. A condição sénior no sul da Europa e na Escandinávia. Saúde e Sociedade, 2015; 24(1): 527-542.

8. CARNEIRO JA, et al. Quedas em idosos não institucionalizados no norte de Minas Gerais: prevalência e fatores associados. Revista Brasileira de Geriatria e Gerontologia, 2016; 19(4): 613-625.

9. EDELMUTH SCVL, et al. Comorbidades, intercorrências clínicas e fatores associados à mortalidade em pacientes idosos internados por fratura de quadril. Revista Brasileira de Ortopedia, São Paulo, 2018; 53(5): 543-551.

10. FALCÃO FRC, et al. Complicações da artroplastia total do quadril em pacientes com e sem diabetes mellitus controlada durante a internação. Revista Brasileira de Ortopedia, São Paulo, 2016; 51(5): 589-596.

11. FARIAS FID, et al. Avaliação da efetividade de um programa de atenção ao idoso com fratura de quadril: uma estratégia de rede. Revista Brasileira Geriatria e Gerontologia, Rio de Janeiro, 2017; 20(5): 705-716.

12. FREITAS MG, et al. Idosos atendidos em serviços de urgência no brasil: um estudo para vítimas de quedas e de acidentes de transito. Ciência \& Saúde Coletiva, Rio de Janeiro, 2015; 20(3): 701-712.

13. FUHRMANN AC, et al. Associação entre a capacidade funcional de idosos dependentes e a sobrecarga do cuidador familiar. Revista Gaúcha de Enfermagem, Porto Alegre, 2015; 36(1): 14-20.

14. GOPINATH B, et al. Hearing and vision impairment and the 5-year incidence of falls in older adults. Age and Ageing. 2016; 45(3): 409-414.

15. GOVEIA VR, et al. Perfil dos pacientes submetidos à artroplastia do quadril em hospital de ensino. Revista do Colégio Brasileiro de Cirurgia, 2015; 42(2): 106-110.

16. GUERRA TEM, et al. Mortalidade em um ano de pacientes idosos com fratura do quadril tratados cirurgicamente num hospital do sul do Brasil. Revista Brasileira de Ortopedia, São Paulo, 2017; 52(1): 17-23.

17. Instituto brasileiro de geografia e estatística. Pesquisa nacional de saúde 2015: Percepção do estado de saúde, estilos de vida e doenças crônicas Brasil, grandes regiões e unidades da federação. Ministério da Saúde. Rio de Janeiro: IBGE; 2016.

18. KERNKAMP CL, et al. Perfil de morbidade e gastos hospitalares com idosos no Paraná, Brasil, entre 2008 e 2012. Cad. Saúde Pública, 2016; 32(7); 1-14.

19. LITWIN H, et al. The complex association between fear of falling and mobility limitation in relation to late-life falls: a share-based analysis. Jounal of Aging and Health, 2018; 30(6): 987-1008.

20. LOURES FB, et al. Análise econômica do tratamento cirúrgico de fratura do quadril em idosos. Revista de Saúde Pública, 2015; 49(12).

21. LOURES FB, et al. Custo-efetividade do tratamento cirúrgico das fraturas de quadril em idosos no Brasil. Revista Brasileira de Ortopedia, São Paulo, 2015; 50(1): 38-42.

22. MATIAS AGC, et al. Análise fatorial de sintomas depressivos e ocorrência de quedas em idosos. Scientia Medica, $2015 ; 25(1): 2-8$.

23. MENTIS M, et al. Psychological and physical problems in elderly people with problems of falls. European Psychiatry, 2017; 41: 659-660.

24. NASCIMENTO JS, TAVARES DMS. Prevalência e fatores associados a quedas em idosos. Texto \& Contexto Enfermagem, 2016; 25(2).

25. OLIVEIRA CC, BORBA VZC. Epidemiologia das fraturas de fêmur em idosos e seu custo para o estado do Paraná, Brasil. Associação Ortopédica Brasileira, 2017; 25(4) 155-158.

26. OLIVEIRA TC, et al. Diferenciais sócio-demográficos da mortalidade de idosos em idades precoces e longevas. Revista Baiana de Saúde Pública, 2015; 39(2): 249-261.

27. PIMENTEL WRT, et al. Quedas e qualidade de vida: associação com aspectos emocionais em idosos comunitários. Geriatrics Gerontology and Aging, 2015; 9(2): 42-48.

28. PRATO SCF, et al. Frequência e fatores associados a quedas em adultos com 55 anos e mais. Revista Saúde Pública, 2017; 51(37).

29. RIZK P, et al. Review of postoperative delirium in geriatric patients undergoing hip surgery. Geriatric Orthopaedic Surgery \& Rehabilitation, 2016; 7(2): 100-105.

30. RODRIGUES GD, et al. Prevenção de quedas no idoso: revisão da literatura brasileira. Revista Brasileira de Prescrição e Fisiologia do Exercício, 2016; 10(59):431-437.

31. SHARMA A, et al. Tratamento de fraturas intertrocanterianas estáveis do fêmur com haste femoral proximal versus parafuso dinâmico do quadril: estudo comparativo. Revista brasileira de Ortopedia, São Paulo, 2018; 53(4): $477-481$.

32. SMITH AA, et al. Assessment of risk of falls in elderly living at home. Revista Latina Americana de Enfermagem, 2017; 25: 2754-2757. 
33. STOLNICKI B, OLIVEIRA LG. Para que a primeira fratura seja a última. Rev. Bras. Ortop., São Paulo, 2016; 51(2): 121-126.

34. TAQUETTE SR, MINAYO MC. Análise de estudos qualitativos conduzidos. Physis Revista de Saúde Coletiva, Rio de Janeiro, 2016; 26(2): 417-434.

35. TYROVOLAS S, et al. Factors associated with skeletal muscle mass, sarcopenia, and sarcopenic obesity in older adults: a multi-continent study. Jounal Cachexia Sarcopenia Muscle, 2016; 7(3): 312-321.

36. VERAS RP, OLIVEIRA M. Envelhecer no Brasil: a construção de um modelo de cuidado. Ciência e Saúde Coletiva, 2018; 23(6): 89-101.

37. VICENTE JRM, et al. Artroplastia total do quadril feita por via posterior minimamente invasiva - resultados após seis anos. Revista Brasileiras Ortopedia, São Paulo, 2015; 50(1): 77-82.

38. WHITEHOUSE MR, et al. Maior mortalidade em 30 dias associada ao uso de hastes intramedulares em comparação com parafusos deslizantes de quadril para o tratamento de fraturas de quadril trocantéricas. Revista Brasileira de Ortopedia, 2019; 101(1). 